\title{
Electrically Controlling the Kondo Effect
}

\author{
Spin-polarized electrons can suppress the experimental signature of the \\ quantum many-body phenomenon known as the Kondo effect.
}

\section{By Marric Stephens}

uke Smith of the National Cheng Kung University in Taiwan and colleagues have demonstrated electrical control of the Kondo effect-a quantum many-body phenomenon first observed in the 1930s [1]. The advance could be a boon for future spintronic devices and spin-based quantum computers, as it promises a more flexible alternative to controlling spin than ferromagnetism-based methods.

The team fabricated a semiconductor heterostructure that hosts a 2D electron gas (2DEG) in a quantum well. On top of the heterostructure, they deposited a pair of metal gates that, when subjected to a voltage, created a 1D constriction in the 2DEG. A potential well at the center of the constriction held a single bound electron.

In such nanostructures, the Kondo effect arises from quantum fluctuations that allow the bound electron to switch places with an oppositely polarized electron in the 2DEG. When both spin-up and spin-down electrons are present in the 2DEG and

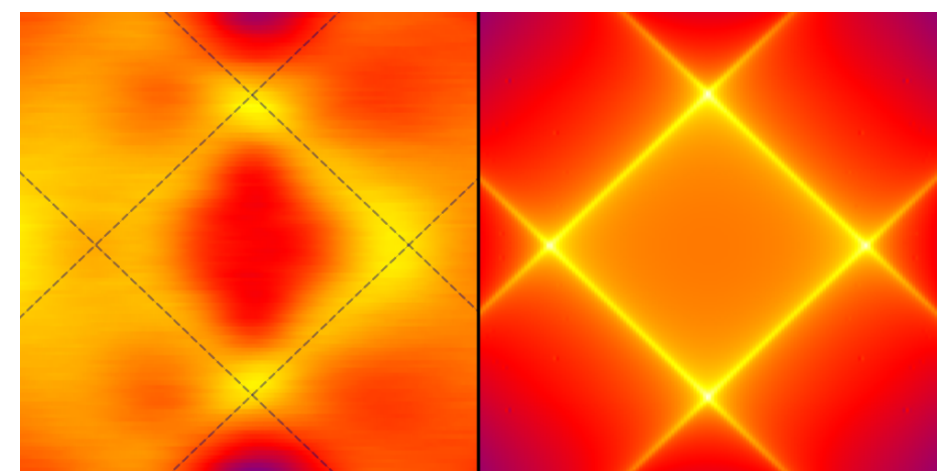

Credit: L. W. Smith et al. [1] no bias is applied, electrons at the Fermi level can flip their spins and tunnel across the constriction. Thus, the effect shows up in measurements as an anomalously high conductance across the 2DEG's constriction.

In the experiment performed by Smith and colleagues, broken inversion symmetry within the heterostructure induced a spin splitting of the 2DEG's energy levels. With an energy separation between spin-up and spin-down electrons, the team found that the zero-bias conductance anomaly-the hallmark of the Kondo effect in this system-no longer occurred. To restore the anomalous conductance, they had to apply a bias to the 2DEG, which realigned the spin-up and spin-down electron energy levels. The team then showed that they could electrically tune the Kondo effect's magnitude by changing the size of the spin splitting via a variable gate voltage.

Marric Stephens is a Corresponding Editor for Physics based in Bristol, UK.

\section{REFERENCES}

1. L. W. Smith et al., "Electrically controllable Kondo correlation in spin-orbit-coupled quantum point contacts," Phys. Rev. Lett. 128, 027701 (2022). 